\title{
Analytical Solutions for Circular Eddies of the Reduced-Gravity, Shallow-Water Equations
}

\author{
Angelo Rubino, Peter Brandt, and Katrin Hessner \\ Institut für Meereskunde, Universität Hamburg, Hamburg, Germany
}

4 March 1997 and 14 July 1997

\begin{abstract}
New analytical, circular eddy solutions of the nonlinear, reduced-gravity, shallow-water equations in a rotating system are presented. While previous analytical solutions were limited to the description of pulsons, which are oscillating, frontal, warm-core eddies with paraboloidic shape and linear velocity components, the new solutions describe more general radial structures of eddy shape and azimuthal velocity. In particular, the new solutions, which contain as a subset the circular pulson solution, also allow for the description of circular, frontal, warmcore eddies with small azimuthal velocities at their periphery and/or with motionless cores, which are frequently observed characteristics of warm-core eddies in the World Ocean.
\end{abstract}

\section{Introduction}

Frontal, warm-core eddies are mesoscale flow features associated with isolated water masses of anomalous water. Thus their properties differ significantly from those of the surrounding ambient water, from which they are separated by a closed frontal line at the sea surface. Such eddies are observed very frequently in the world ocean, the most famous example being perhaps the warm-core rings released by the meanders of the Gulf Stream (Cheney et al. 1976; Evans et al. 1984; Joyce 1984). The influence of frontal, warm-core eddies on the larger-scale ocean circulation involves the transfer of energy and physical, chemical, and biological properties across frontal zones and their impact on mixing (Olson 1991). This relevance of frontal, warm-core eddies explain why, in the last two decades, many analytical, numerical, experimental, and observational investigations have been carried out to elucidate different aspects of their dynamics (e.g., Saunders 1971; Olson et al. 1985; Pavia and Cushman-Roisin 1988; Rogers 1989; Pavia and Lopez 1994; Matsuura 1995; CushmanRoisin and Merchant-Both 1995).

In the study of frontal, warm-core eddies, the reducedgravity, shallow-water equations have been widely used (Csanady 1979; Nof 1983; Killworth 1983; CushmanRoisin et al. 1985; Cushman-Roisin 1987; Arai et al. 1994). Although these equations exclude relevant oceanic processes such as baroclinic instabilities, they rep-

Corresponding author address: Dr. Angelo Rubino, Institut für Meereskunde, Universität Hamburg, Troplowitzstr. 7, D-22529 Hamburg, Germany.

E-mail: rubino@ifm.uni-hamburg.de resent an efficient tool for the description of many characteristics of eddy dynamics. Moreover, an exact analytical solution of these equations has been found, which describes the temporal and spatial evolution of a special class of frontal, warm-core eddies, the so-called pulsons, characterized by a paraboloidic shape and by horizontal velocities that are linear functions of the horizontal coordinates (Thacker 1981; Cushman-Roisin 1987; Rogers 1989). Actually, observed frontal, warm-core eddies often differ remarkably from the pulson either in their velocity field or in their shape (Richardson et al. 1973; Andrews and Scully-Power 1976; Evans et al. 1985 and references therein). In the present paper we propose an extension of the circular pulson solution that can be used to describe more general radial structures of eddy shape and azimuthal velocity. The paper is organized as follows: In section 2 the reduction of the problem to a set of series of ordinary differential equations in time is presented. The general analytical solutions of the problem are given in section 3, and, in section 4, these solutions are discussed and conclusions are presented.

\section{Reduction of the problem to a system of ordinary differential equations}

Assuming circular symmetry, the nonlinear, reducedgravity, shallow-water equations for a rotating system expressed in polar coordinates are

$$
\begin{array}{r}
\frac{\partial v_{\vartheta}}{\partial t}+v_{r} \frac{\partial v_{\vartheta}}{\partial r}+\frac{v_{r} v_{\vartheta}}{r}+f v_{r}=0, \\
\frac{\partial v_{r}}{\partial t}+v_{r} \frac{\partial v_{r}}{\partial r}-\frac{v_{\vartheta}^{2}}{r}-f v_{\vartheta}+g^{\prime} \frac{\partial h}{\partial r}=0,
\end{array}
$$




$$
\frac{\partial h}{\partial t}+\frac{\partial h v_{r}}{\partial r}+\frac{h v_{r}}{r}=0 .
$$

Here $h$ is the thickness of the upper layer, $f$ is the (constant) Coriolis parameter, $g^{\prime}$ is the reduced gravity, $t$ is the time, and $v_{\vartheta}$ and $v_{r}$ represent the azimuthal and radial velocity components of the upper layer along the $\vartheta$ and $r$ coordinates. Positive $v_{r}$ are directed from the coordinate origin, located at the eddy center, toward the eddy periphery, and positive $v_{\vartheta}$ are directed counterclockwise.

Solutions of (1)-(3) are searched that represent circular, frontal, warm-core eddies with the following velocity field and shape:

$$
\begin{aligned}
v_{\vartheta} & =-\sum_{i=1}^{n} L_{i} r^{2 i-1} \\
v_{r} & =K r \\
h & =\sum_{i=0}^{2 n-1} A_{i} r^{2 i}
\end{aligned}
$$

where the coefficients $L_{i}, K$, and $A_{i}$ are functions of time only and $n \geq 1$ is the order of the system. Inserting (4)-(6) into (1)-(3) yields the following series of ordinary differential and algebraic equations:

$$
\begin{aligned}
& \frac{d L_{i}}{d t}+2 i K L_{i}-\delta_{i 1} f K=0, \quad i=1, \cdots, n \\
& \delta_{i 1} \frac{d K}{d t}-\sum_{j=1}^{i} L_{j} L_{i-j+1}+\delta_{i 1} K^{2}+f L_{i}+2 i g^{\prime} A_{i}=0, \quad i=1, \cdots, 2 n-1 \\
& \frac{d A_{i}}{d t}+2(i+1) K A_{i}=0, \quad i=0, \cdots, 2 n-1,
\end{aligned}
$$

where $\delta_{i 1}$ is the Kronecker delta and $L_{i}=0$ for $i>n$.

For $n=1$, this set of series yields the four ordinary differential equations that describe the circular pulson:

$$
\begin{aligned}
\frac{d L_{1}}{d t}+2 K L_{1}-f K & =0 \\
\frac{d K}{d t}-L_{1}^{2}+K^{2}+f L_{1}+2 g^{\prime} A_{1} & =0 \\
\frac{d A_{0}}{d t}+2 K A_{0} & =0 \\
\frac{d A_{1}}{d t}+4 K A_{1} & =0 .
\end{aligned}
$$

From (10)-(13) the coefficients $L_{1}, K, A_{0}$, and $A_{1}$ can be determined (Cushman-Roisin 1987). This system of equations has four degrees of freedom. Every successive order $m$ introduces in the system three additional ordinary differential equations [(7) with $i=m$ and (9) with $i=2 m-2$ and $i=2 m-1]$ and two algebraic equations [(8) with $i=2 m-2$ and $i=2 m-1]$. Thus, due to these two algebraic equations, only one degree of freedom is added to the system for every successive order. Note that Eq. (8) for $i>1$ represent the set of conditions relating $L_{i}(1 \leq i \leq m)$ and $A_{i}(2 \leq i \leq 2 m$ $-1)$. It can be shown that, if these conditions are satisfied initially, then they will be satisfied for every time.

Before presenting the general solutions of (7)-(9), we have to note that the description of a circular eddy whose fluid circulates inside of a surface frontal line requires that the following conditions are satisfied: (i) $h(r)=0$ for $r=R$, where $R>0$ is the time-dependent eddy surface radius and (ii) $h(r)>0$ for $r<R$. It can be shown that, if conditions (i) and (ii) are satisfied initially, then they will be satisfied for every time. Thus the solutions of (10)-(13) are valid at all times for $r \leq R$.

\section{General solutions}

The general solutions of (7)-(9) are

$$
\begin{gathered}
L_{i}=\delta_{i 1} \frac{f}{2}+\frac{\tilde{L}_{i}}{[1+\gamma \sin (f t+\varphi)]^{i}}, \\
i=1, \cdots, n, \\
K=\frac{f \gamma}{2} \frac{\cos (f t+\varphi)}{[1+\gamma \sin (f t+\varphi)]}, \\
A_{i}=\frac{\tilde{A}_{i}}{[1+\gamma \sin (f t+\varphi)]^{i+1}}, \\
i=0, \cdots, 2 n-1,
\end{gathered}
$$

where $\gamma, \varphi$, and $\tilde{A}_{0}$ are arbitrary constants of integration. The range of applicability of these parameters is $0 \leq \gamma$ $<1,0 \leq \varphi<2 \pi$, and $\tilde{A}_{0}>0$. The last condition is required for the description of eddies whose fluid circulates inside of a surface frontal line. The coefficients $\tilde{A}_{i}$ and $\tilde{L}_{i}$ are related to each other by 


$$
\begin{gathered}
2 i g^{\prime} \tilde{A}_{i}=-\delta_{i 1} \frac{f^{2}\left(1-\gamma^{2}\right)}{4}+\sum_{j=1}^{i} \tilde{L}_{j} \tilde{L}_{i-j+1}, \\
i=1, \cdots, 2 n-1,
\end{gathered}
$$

with $\tilde{L}_{i}=0$ for $i>n$. Together with conditions (17), the solutions of (1)-(3), which describe circular, frontal, warm-core eddies, are

$$
\begin{aligned}
v_{\vartheta} & =-\frac{f}{2} r-\sum_{i=1}^{n} \tilde{L}_{i} \frac{r^{2 i-1}}{[1+\gamma \sin (f t+\varphi)]^{i}}, \\
v_{r} & =\frac{f \gamma}{2} \frac{\cos (f t+\varphi)}{[1+\gamma \sin (f t+\varphi)]} r, \\
h & =\sum_{i=0}^{2 n-1} \tilde{A}_{i} \frac{r^{2 i}}{[1+\gamma \sin (f t+\varphi)]^{i+1}} .
\end{aligned}
$$

In analogy to the circular pulson solution (CushmanRoisin 1987), these solutions describe oscillating, circular eddies that alternatively contract and deepen, expand and shoal during an (exact) inertial period $T=$ $2 \pi / f$.

\section{Discussion}

The new analytical solutions of the nonlinear, reduced-gravity, shallow-water equations (1)-(3) given by (17)-(20) can be used to describe the temporal and spatial evolution of frontal, warm-core eddies. While they represent an extension of the circular pulson solution (Cushman-Roisin 1987) to more general radial structures of eddy shape and azimuthal velocity, they retain the radial velocity structure of the circular pulson. This implies that the temporal evolution of the eddy center depth and radius remains unchanged, independent of the order of the solution. However, frontal, warm-core eddies whose azimuthal velocities even largely differ from a linear function of the radius and/or whose shapes even largely differ from a paraboloid exist as analytical solutions of (1)-(3). One example is depicted in Fig. 1. Here, the depth of the interface separating the eddy from the ambient water (Fig. 1a), the azimuthal (Fig. 1b), and the radial velocity components of the eddy (Fig. 1c) are shown as functions of the radius for four different times of an inertial period. In this case the maximum steepness of the interface depth is not located at the eddy periphery as it would be in the case of the pulson. Accordingly, the associated azimuthal velocity decreases from its maximum, located inside the eddy, toward the periphery where it is almost zero (Fig. 1b). Thus, due to this characteristic of our solutions, a more realistic description of eddy dynamics is possible than is possible using the previous circular pulson solution (Cushman-Roisin 1987), which predicts unrealistically large azimuthal velocities at the eddy periphery. Furthermore, a central region (core) of the eddy exists where the interface depth is almost constant (Fig. 1a). Accordingly, the associated azimuthal velocity is, in this region, almost zero (Fig.
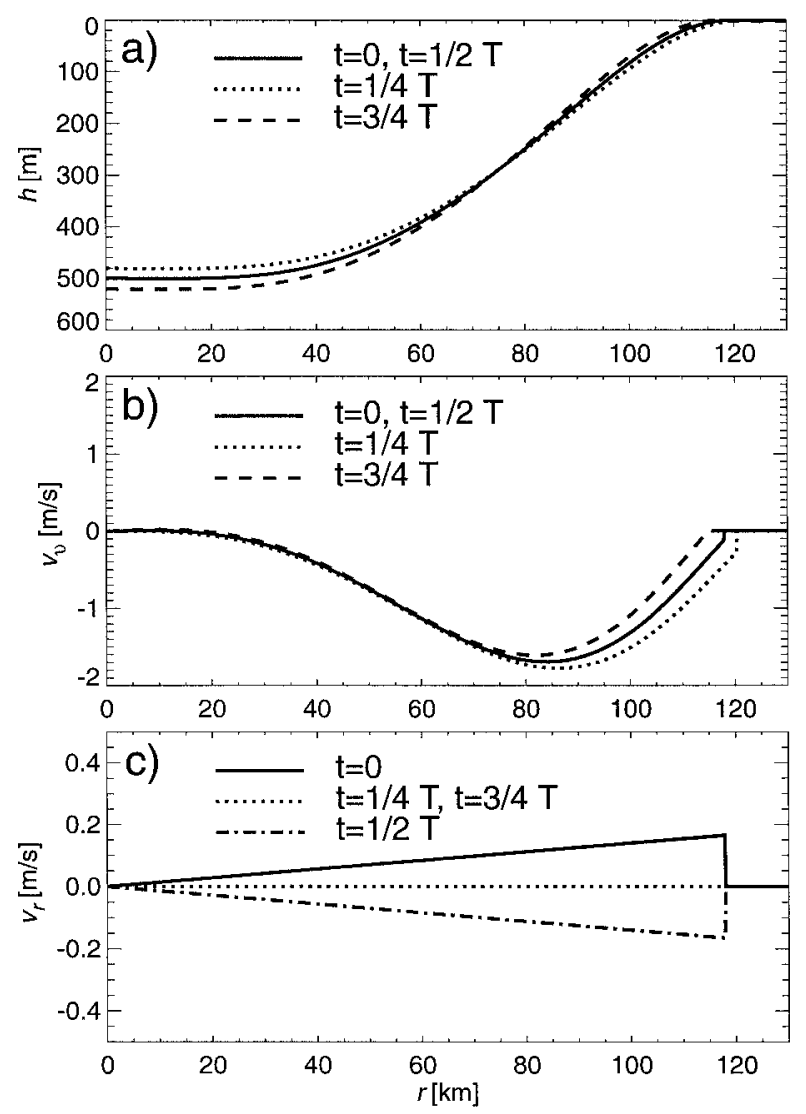

FIG. 1. Interface depth $h$ (a), azimuthal velocity component $v_{\vartheta}$ (b), and radial velocity component $v_{r}$ (c) of a circular, frontal, warm-core eddy for the times $t=0, t=(1 / 4) T, t=(1 / 2) T$, and $t=(3 / 4) T(T=$ $2 \pi / f)$ as function of the radius $r$. The following values for the parameters of (17)-(20) have been used: $n=4, f=7 \times 10^{-5} \mathrm{~s}^{-1}, g^{\prime}$ $=10^{-2} \mathrm{~m} \mathrm{~s}^{-2}, \tilde{A}_{0}=500 \mathrm{~m}, \gamma=0.04, \varphi=0, \tilde{L}_{1}=-1.492 \times 10^{-6}$ $\mathrm{s}^{-1}, \tilde{L}_{2}=9.169 \times 10^{-15} \mathrm{~m}^{-2} \mathrm{~s}^{-1}, \tilde{L}_{3}=-1.088 \times 10^{-24} \mathrm{~m}^{-4} \mathrm{~s}^{-1}$, and $\tilde{L}_{4}=3.177 \times 10^{-35} \mathrm{~m}^{-6} \mathrm{~s}^{-1}$.

1b). This characteristic of our solutions corresponds to a frequently observed characteristic of frontal warmcore eddies that also cannot be described by the pulson solution. Note, however, that the radial velocity is, as in the case of the circular pulson, a linear function of the radius (Fig. 1c). The temporal evolution of the eddy can be described as follows: At $t=0$ its radial velocity is divergent and, consequently, the eddy expands and shoals. At $t=(1 / 4) T$ the eddy is shallowest. At this time its azimuthal velocity is maximum, while its radial velocity vanishes. At $t=(1 / 2) T$ the radial velocity of the eddy is convergent and, consequently, the eddy contracts and deepens. At $t=(3 / 4) T$ the eddy is deepest. At this time its azimuthal velocity is minimum, while its radial velocity vanishes again.

The presented solutions can also be used to infer the internal structure of frontal, warm-core eddies from surface measurements. For example, remote sensing techniques give the possibility to measure the spatial extent as well as the velocity structure of frontal eddies at the 
sea surface. If this information is provided, the coefficient $\tilde{L}_{i}$ can be calculated by fitting the measured velocity by a polynomial. Thus, using (17), the coefficients $\tilde{A}_{i}(i \geq 1)$ can be calculated, which describe the spatial structure of the eddy. The center depth $\tilde{A}_{0}$ can thus be obtained by letting the measured and calculated eddy surface radius coincide. In this way, the whole stationary structure of the eddy can be determined. A sequence of remote sensing measurements could then be used to estimate the eddy pulsation in time.

Acknowledgments. This work was partly funded by the German Space Agency (DARA) under Contract 50EE9413. The authors would like to thank Stefano Pierini, Dmitri Sein, Juan Heberto Gaviño-Rodriguez, and Werner Alpers for helpful discussions.

\section{REFERENCES}

Andrews, J. C., and P. Scully-Power, 1976: The structure of an East Australian current anticyclonic eddy. J. Phys. Oceanogr., 6, 756765 .

Arai, M., and T. Yamagata, 1994: Asymmetric evolution of eddies in rotating shallow water. CHAOS, 4, 163-175.

Cheney, R. E., W. H. Gemmill, and M. K. Shank, 1976: Tracking a Gulf Stream ring with SOFAR floats. J. Phys. Oceanogr., 6, 741-749.

Csanady, G. T., 1979: The birth and death of a warm core ring. $J$. Geophys. Res., 84, 777-780.

Cushman-Roisin, B., 1987: Exact analytical solution for elliptical vortices of the shallow-water equations. Tellus, 39A, 235-244. , and S. Merchant-Both, 1995: Elliptical warm core rings in a two-layer ocean with ambient shear. J. Phys. Oceanogr., 25, 2011-2024.

- W. H. Heil, and D. Nof, 1985: Oscillations and rotations of elliptical warm-core rings. J. Geophys. Res., 90, 11 756-11 764.

Evans, R. H., K. S. Baker, O. B. Brown, R. C. Smith, S. Hooker, D. Olson, and the Warm-Core-Rings Program Service Office, 1984: Satellite images of warm-core ring 82-B, sea surface temperature and a chronological record of major physical events affecting ring structure. Woods Hole Oceanographic Institution Rep., 25 pp. [Available from Woods Hole Oceanogr. Inst., Woods Hole, MA, 02543.]

$-,-\longrightarrow,-$, and -1985 : Chronology of warm-core ring 82B. J. Geophys. Res., 90, 8803-8811.

Joyce, T. M., 1984: Velocity and hydrographic structure of a warmcore ring. J. Phys. Oceanogr., 14, 936-947.

Killworth, P. D., 1983: On the motion of isolated lenses on a betaplane. J. Phys. Oceanogr., 13, 368-376.

Matsuura, T., 1995: The evolution of frontal-geostrophic vortices in a two-layer ocean. J. Phys. Oceanogr., 25, 2298-2318.

Nof, D., 1983: On the migration of isolated eddies with application to Gulf Stream rings. J. Mar. Res., 41, 399-425.

Olson, D. B., 1991: Rings in the ocean. Annu. Rev. Earth Planet. Sci., 19, 283-311.

— - R. W. Schmitt, M. Kennelly, and T. M. Joyce, 1985: A twolayer diagnostic model of the long-term physical evolution of warm-core ring 82B. J. Geophys. Res., 90, 8813-8822.

Pavia, E. G., and B. Cushman-Roisin, 1988: Modeling of oceanic fronts using a particle method. J. Geophys. Res., 93, 3554-3562.

- , and M. Lopez, 1994: Long-term evolution of elongated warm eddies. J. Phys. Oceanogr., 24, 2201-2208.

Richardson, P. L., A. E. Strong, and J. A. Knauss, 1973: Gulf Stream eddies: Recent observations in the western Sargasso Sea. J. Phys. Oceanogr., 3, 297-301.

Rogers, C., 1989: Elliptic warm-core theory: The pulsrodon. Phys. Lett., 138, 267-273.

Saunders, P. M., 1971: Anticyclonic eddies formed from shoreward meanders of the Gulf Stream. Deep-Sea Res., 18, 1207-1220.

Thacker, W. C., 1981: Some exact solutions to the non-linear shallowwater wave equations. J. Fluid Mech., 107, 499-508. 